\title{
LPWAN Technologies for IoT Systems: Choice Between Ultra Narrow Band and Spread Spectrum
}

\author{
Nitin Naik \\ Defence School of Communications and Information Systems \\ Ministry of Defence, United Kingdom \\ Email: nitin.naik100@mod.gov.uk
}

\begin{abstract}
Low Power Wide Area Network (LPWAN) is imperative for the expansion and development of IoT networks and their connectivity infrastructure. This far-reaching connectivity of low power devices that are placed virtually anywhere is evolving a new things-based business model. This things-based business model has certain requirements such as long range, extended battery life and very low end point cost. LPWAN technologies have successfully addressed these IoT requirements and are receiving wider acceptance in the IoT industry. In most LPWAN technologies, two main alternative communication techniques, Ultra Narrow Band (UNB) and Spread Spectrum (SS) are used at the physical layer. However, the greatest dilemma is the selection of the most suitable technique from UNB and SS for LPWAN. This paper addresses this selection dilemma of UNB and SS by examining some of the most critical factors responsible for the performance of LPWAN technologies such as interference, capacity, link budget and coexistence. Furthermore, it evaluates the most popular UNB-based LPWAN technologies Sigfox and Telensa, and SSbased LPWAN technologies LoRa and RPMA investigating their strengths and limitations for IoT applications.
\end{abstract}

Index Terms-LPWAN; Low Power Wide Area Network; IoT System; M2M; Ultra Narrow Band; UNB; Spread Spectrum; SS; LoRA; RPMA; Sigfox; Telensa.

\section{INTRODUCTION}

Low Power Wide Area Network (LPWAN) covers a range of technologies that are used to connect low power devices such as sensors and controllers in Internet of Things (IoT) and Machine-To-Machine (M2M) communications. IoT and M2M communications require a wireless sensor network with certain requirements such as long range, extended battery life and very low end point cost [1]. LPWAN technologies have successfully fulfilled all these requirements of IoT and M2M communications. Therefore, they are getting wider acceptance in the IoT industry. Machina Research has predicted about 1.4 billion LPWAN connections by 2022 [2], thus LPWAN will be exceeding $2 \mathrm{G}, 3 \mathrm{G}$ and $4 \mathrm{G}$ connections and becoming the leading technology for the IoT and M2M connectivity [3].

In most LPWAN technologies, two main alternative communication techniques, Ultra Narrow Band (UNB) and Spread Spectrum (SS) are used at the physical layer. UNB technique is used to transmit a signal with a very small bandwidth and mostly suited for the small uplink traffic [4]. This old UNB technique has been revived for IoT and M2M communications again due to the advancement in signal processing technology. In UNB, a signal is modulated using abrupt phase shift modulation [5]. Subsequently, it is processed through a UNB filter to filter all sidebands and harmonics for keeping a single sideband [6]. SS technique is one of the oldest communication technique used in military applications for secure communications by spreading the original signal over a large frequency band. SS technique uses additional bandwidth than the actual signal, though it preserves the same signal power [7]. It has no noticeable peak in the spectrum, which resembles with noise, and therefore, it is difficult to jam or intercept [8], [9].

Both UNB and SS fulfil the requirements of the long range coverage with long battery life for industrial IoT systems. However, UNB and SS are quite different techniques including the most obvious difference of the use of ultra narrow band and wideband respectively. Both have their own strengths and limitations for the IoT/M2M connectivity. This paper addresses this selection dilemma of UNB and SS by examining some of the most critical factors responsible for the performance of LPWAN technologies such as interference, capacity, link budget and coexistence. Furthermore, it evaluates the most popular UNB-based LPWAN technologies Sigfox and Telensa, and SS-based LPWAN technologies LoRa and RPMA investigating their strengths and limitations for IoT applications.

The rest of the paper is organised as follows: Section II elucidates about LPWAN, its suitability for IoT and M2M, characteristics and classification; Section III elucidates about Ultra Narrow Band and Spread Spectrum techniques; Section IV performs the evaluation of UNB and SS for LPWAN technologies based on the interference, capacity, link budget and coexistence; Section V expounds the comparative analysis of UNB-based LPWAN technologies Sigfox and Telensa, and SS-based LPWAN technologies LoRa and RPMA; Section VI concludes the paper and suggests the future work.

\section{LOW POWER Wide AREA NETWORK (LPWAN)}

\section{A. What is LPWAN?}

The term Low Power Wide Area Network (LPWAN) is formed from conjoining two phrases Low Power (consumption of low power) and Wide Area Network (long range of communication), describing its two intrinsic characteristics small power budget (i.e., longer battery life) and extended range of transmission. These characteristics of 
LPWAN including for example, low cost and low data rate, make it a unique communication technology for wireless communications and a preferred choice for IoT and M2M applications. While LPWAN is not a single technology, it encompasses a range of technologies, which are used to connect low power devices such as sensors and controllers in IoT and M2M communications. LPWAN technology can operate on either the licensed or unlicensed spectrum and comprise of proprietary, alliance or open standard options. Network designers have utilised LPWAN technologies to design bespoke wireless sensor networks and customise the cost, coverage and power consumption of transmission for specific IoT and M2M applications.

\section{B. Why LPWAN is well-suited for IoT and M2M?}

Today, several wireless communication technologies are available to design a wireless network, however, the majority are not entirely suitable for IoT and M2M communications with regards to data rate, range of communication and power consumption (battery life). As shown in Fig. 1, some wireless communication technologies provide a higher data rate, long range with higher power requirement (e.g., cellular LTE, WiMax); some communication technologies provide a lower data rate, short range with lower power requirement (e.g., Bluetooth/BLE); or some communication technologies provide a higher data rate, short range with higher power requirement (e.g., WiFi). Whereas most IoT and M2M solutions require a wireless sensor network providing a lower data rate, long range with lower power requirement (longer battery life) [10], [11], which is not completely satisfied by the existing wireless communication technologies as explained earlier. LPWAN technologies are designed to fulfil these requirements, and therefore, they are well-suited for IoT and M2M communications that send small amounts of data over a long range, while maintaining long battery life. Additionally, many LPWAN technologies perform well in obstructed environments such as in cities and rugged outdoor environments.

\section{Characteristics of LPWAN}

The main characteristics of LPWAN technologies required to successfully support commercial deployment of IoT and M2M systems are:

- Long range of communication

- Low power consumption

- Low data rate

- Low cost of device and deployment

- Simplified network topology and deployment

- Full coverage (improved outdoor and indoor penetration coverage)

- Network scalability for the capacity upgrade

\section{Classification of LPWAN}

In IoT and M2M communications, the choice of a LPWAN technology is dependent on the specific application area and its specific requirements such as data rate, range, energy budget, frequency band, bidirectionality, network

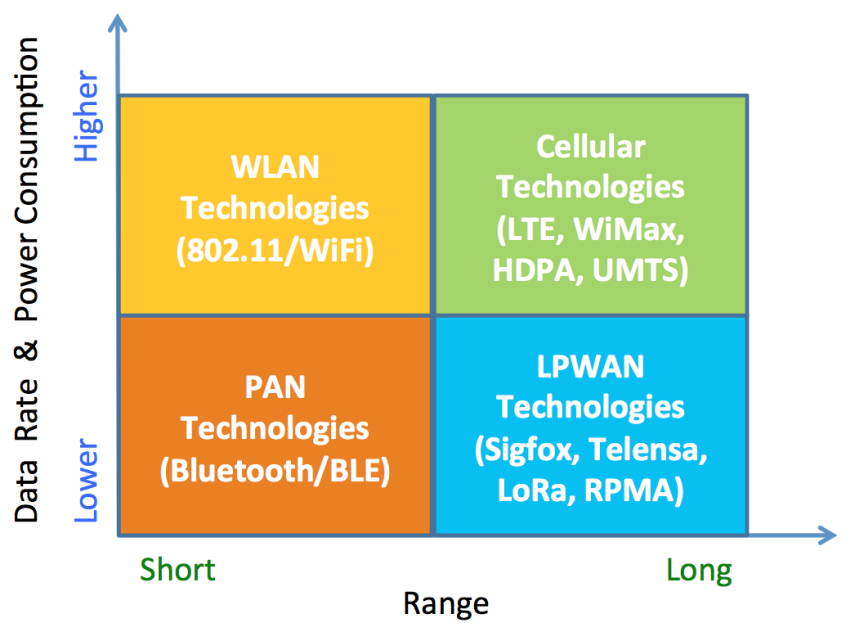

Fig. 1: Comparison of Data Rate, Power Consumption and Range for Wireless Communication Technologies

cost, scalability and security. While there are many LPWAN technologies, they can be broadly classified into two categories: licensed LPWAN technologies (e.g., NBIoT, LTE-M-IoT, EC-GSM-IoT and 5G IoT) and licence exempt or unlicensed LPWAN technologies (e.g., Sigfox, Telensa, LoRa and RPMA). The majority of these LPWAN technologies use two main alternative approaches to support the physical layer communications Ultra Narrow Band (UNB) and Spread Spectrum (SS). In addition to choosing the licensed and unlicensed LPWAN, it is crucial to understand the advantages and disadvantages of UNB and SS in selecting which meets the requirements for a specific IoT/M2M application. These points are discussed in the rest of the paper.

\section{Ultra NARROW BAND (UNB) AND SPREAD SPECTRUM (SS) TECHNIQUES}

\section{A. Ultra Narrow Band (UNB)}

Ultra Narrow Band employs an ultra-narrow spectrum channel $(<1 \mathrm{KHz})$ to establish an ultra-long distance link between transmitter and receiver. It offers superb link budget due to the concentration of power in a narrow frequency band and low in-band receive noise (narrow receive filters remove most of the noise) [4], [12]. Consequently, it allows the long range coverage with long battery life (lower transmit power), which makes it a most suitable LPWAN technique for industrial IoT systems. Additionally, its ultra-high power spectral density (PSD) creates endurance against interference and jamming, which enables friendly coexistence of UNB on shared frequency bands [4], [13]. Many traditional modulation approaches require allowance for upper and lower sidebands throughout the carrier frequency. UNB modulation is a modified approach for data transmission without much relying on its sidebands [14]. 


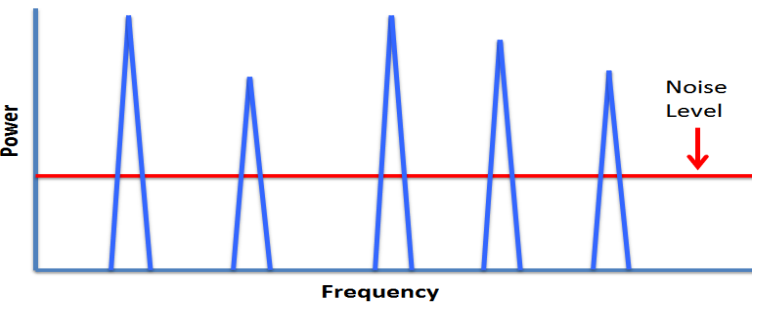

Fig. 2: Ultra Narrow Band (UNB) Signals

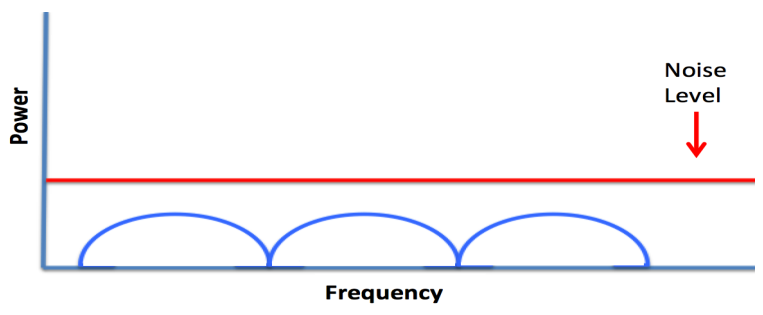

Fig. 3: Spread Spectrum (SS) Signals

\section{B. Spread Spectrum (SS)}

Spread Spectrum is an alternative technique to UNB for industrial IoT systems, which employs wideband (noise-like signals) to transmit the data and spread the data signal over a bandwidth which is much larger than the bandwidth of an actual data signal. Unlike in narrowband, where data is transmitted through a single RF band; whereas in SS, data is transmitted by changing its carrier frequencies alternatively or the pattern of data continuously. SS transmitters operate at the same transmit power level to narrowband transmitters. This is because of SS signals are wide, therefore, they can transmit at a lower spectral power density $(\mathrm{W} / \mathrm{Hz})$ in comparison to narrowband transmitters. This is one of the biggest advantage of SS and its popularity for low power IoT devices. Also, SS signals are difficult to detect, intercept, demodulate and jam. SS technique can be classified into several categories such as DSSS, FHSS and CSS.

\section{EVALUATION OF UNB AND SS IN LPWAN TECHNOLOGIES FOR IOT SYSTEMS}

\section{A. Interference}

UNB technology employs narrow RF channels and neighbouring sub-carriers can be orthogonal, which avoids interference between sub-carriers and distributes the power among the sub-carriers to compensate the fading environment of each carrier. UNB for LPWAN applications mainly employs one sub-carrier for the uplink from each endpoint and the base station can process multiple uplink sub-carriers, which is very similar to a wideband system that uses multiple sub-carriers. The utilisation of multiple narrowband sub-carriers decreases the number of end-points sharing access to a given channel to lessen the chance of interference with other narrowband users. The noise level encountered by a one narrowband in UNB is also minimal as shown in Fig. 4. Consequently, the receiver does not need processing gain via frequency de-spreading for decoding

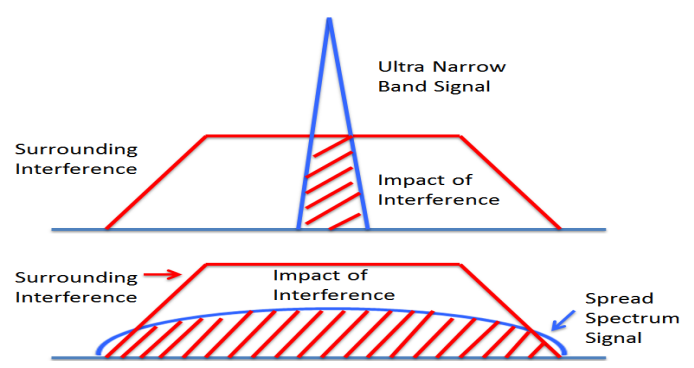

Fig. 4: Interference impact on UNB and SS signals

the signal, this facilitates simple and inexpensive transceiver design [14]. Nonetheless, in UNB, small frequency changes over time may become comparatively significant with respect to the signal bandwidth. These drifts in frequency cause several negative impacts such as difficult to detect and demodulate the signal and increasing the likelihood of collision between messages.

$\mathrm{SS}$ is a wideband technology (nonetheless the reverse is not true) and offers several benefits including resistance to interference and jamming. Most interference (narrowband or wideband) and jamming signals are rejected because they do not have the SS key used in the de-spreading process at receiver [15]. All SS have a threshold/tolerance limit to interference and communication stops beyond this limit. This threshold limit is determined by SS processing gain that is the ratio of the RF bandwidth to the information bandwidth [16]. Despite all these benefits, SS can be saturated swiftly and may have further interference issues such as self-noise in dense environments. The reason of self-noise is that each device/user is a source of interference for other devices/users. Additionally, it is essential that the receiver obtains the equal power from each transmitter and if it receives extra power from any device/user, this means that a device/user is causing further interference to other devices/users. The power control guarantees that they are received with the equal power $P_{R X}$ at the receiver to avoid interference [17], [18].

Summary: In a nutshell, in LPWAN, the range is decided based on the data rate. Thus, the lower data rate delivers longer range owing to the increased sensitivity for the receiver. However, it is a trade-off between range and the transmission time because lower data rate will take longer transmission time for packets, which will increase the possibility of interference/collisions with other LPWAN networks [13]. Generally, UNB offers lower data rate than SS, therefore this slow data rate and frequency drifting issue of UNB may increase the possibility of interference in UNBbased LPWAN. Otherwise, the possibility of interference in SS is higher than UNB (see Fig. 5) because of the use of wideband and implementation loss cannot completely offset by the processing gain (the decrease in signal-to-noise ratio is offset by the processing gain) [19]. 

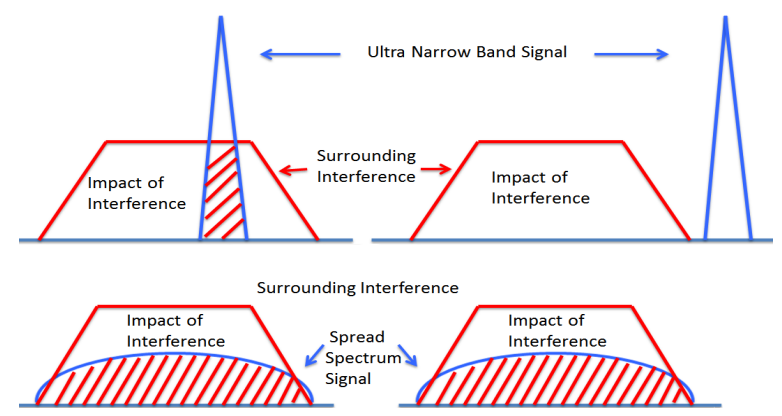

Fig. 5: Interference impact on UNB and SS signals

\section{B. Capacity}

In LPWAN technologies, coverage determines the range/area for the effective transmission of data, while capacity empowers us to perform various operations using that link. Capacity is an exploitable throughput (the actual amount of data) over on link after subtracting MAC data and all the other overheads including security, interference and all real-world expenses. However, a data rate is a PHY layer metric, which signifies the overall data but not the actual throughput experienced by user. Capacity is also different for an uplink and downlink. The capacity of standard Additive White Gaussian Noise (AWGN) channel for UNB and SS techniques can be evaluated based on the Shannon channel capacity. Here, it is defined in the form of overall bit rate for all users per unit of available bandwidth. The Shannon channel capacity formula for the maximum theoretical data rate $C$ (bps) for bandwidth $B(\mathrm{~Hz})$ and received power $P_{R X}(\mathrm{~W})$ for a single user is:

$$
C=B \log _{2}\left(1+\frac{P_{R X}}{B N_{0}}\right)
$$

Where $N_{0}$ is the noise density $(\mathrm{W} / \mathrm{Hz})$, and $B N_{0}$ is the noise power $(\mathrm{W})$ in the channel bandwidth.

The capacity of UNB can be calculated based on pure FDMA system, where the overall bandwidth can be divided among $k$ users with a received power $P_{R X}$ and a bandwidth of $B / k$. Thus, the system capacity available to $k$ users is:

$$
C_{k}=B \log _{2}\left(1+\frac{k P_{R X}}{B N_{0}}\right)
$$

Similarly, the capacity of SS can be calculated for $k$ users based on an assumption of the same SNR requirement (i.e. perfect power control in the uplink) for each user:

$$
C_{k}=B \log _{2}\left(1+\frac{P_{R X}}{B N_{0}+(k-1) P_{R X}}\right)
$$

Based on the above formula, system capacity will increase in the UNB technique if the channel is partitioned into further smaller frequency segments assuming that no constraint is on the power flux density that can be utilised. Thus, UNB technique may have higher channels than SS in the same bandwidth, which is also shown in Fig. 6. However, UNB needs complex signal processing and extra

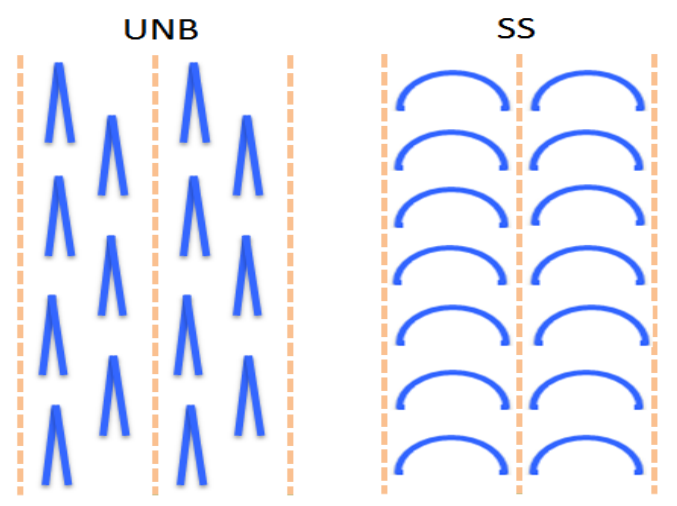

Fig. 6: Comparison of capacity of UNB and SS

synchronization at receiver. In the SS technique, the optimised use of the spectrum is relatively difficult because of the addition of the redundant data in the coding to offset the higher noise floor [13]. Moreover, an increasing number of users sharing the channel will cause extra noise to other users and eventually, this self-noise situation will limit the overall network capacity. Additionally, working with lower fade margins is one of the main advantage in SS, but in LPWAN, it is not even helpful. In low-level of bi-directional communications, the superior power control is a challenging task. Theoretically, the superior power control in multi-user detection SS will permit the same capacity as UNB but at the cost of greater complexity.

Summary: In a nutshell, in LPWAN, it is difficult to achieve the optimised condition in SS network, which can provide the same capacity as the UNB network. Additionally, an extra data is added in the SS coding to offset the higher noise floor, which reduces the capacity of SS [13]. Therefore, UNB technique can support greater capacity as compared to SS technique with comparatively less complex system. Theoretically, in an isolated environment, a UNB network may have nearly five times the uplink capacity of a SS network [20]. However, the actual capacity of UNB may be considerably lower than the theoretical capacity due to functional restrictions of base station receivers [21].

\section{Link Budget}

A link budget can be considered as a single metric to compare any two wireless technologies. A higher link budget signifies the better coverage for that particular wireless technology. The link budget includes all the parameters that will decide the strength of an arriving signal at the receiver. It is an accounting of all the possible gains and losses in any transmission system (between the transmitter and the receiver). This includes transmitter power, antennas gain, path loss, propagation loss, cable loss, modulation choices, receiver sensitivity and other system dependent gains and losses. LPWAN is a constrained network, therefore, the link budget is the most crucial factor to design optimised and customised IoT system to deliver the desired functionalities successfully without being over designed at extra cost. Most 


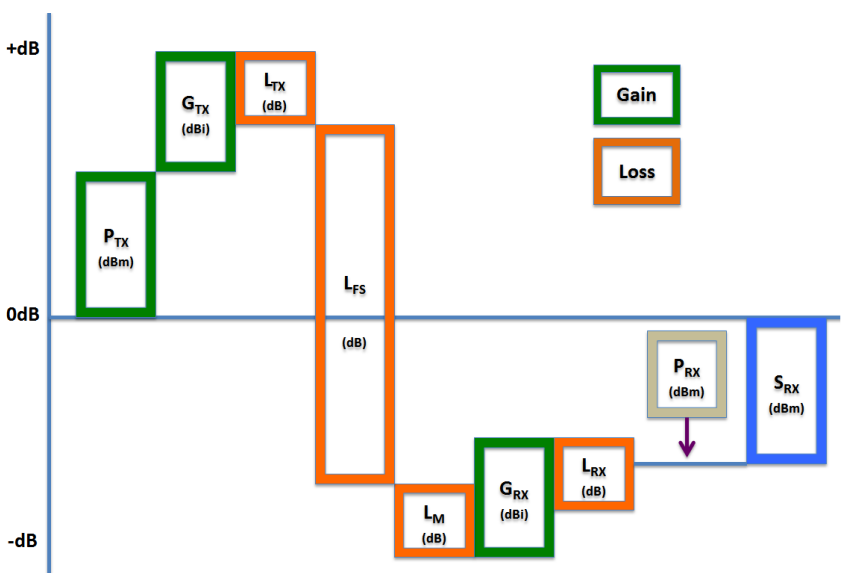

Fig. 7: Parameters used in computation of the link budget for UNB and SS

LPWAN solutions link budget is in the range of $156 \mathrm{~dB}$ up to $172 \mathrm{~dB}$, where a common value is around $160 \mathrm{~dB}$. The link budget of LPWAN can be calculated as given in Equation:

Received Power $(\mathrm{dBm})=$ Transmitted Power $(\mathrm{dBm})$

$$
+ \text { Gains }(\mathrm{dB}) \text { - Losses }(\mathrm{dB})
$$

The above Equation 4 can be described in detail with corresponding parameters as given in Equation 5:

$$
P_{R X}=P_{T X}+G_{T X}+G_{R X}-L_{T X}-L_{F S}-L_{M}-L_{R X}
$$

Finally, the link budget yields System Operating Margin (SOM) as shown in Equation 6:

$$
\text { System Operating Margin }=P_{R X}-S_{R X}
$$

Where:

- $P_{R X}=$ Received power $(\mathrm{dBm})$

- $P_{T X}=$ Transmitter output power $(\mathrm{dBm})$

- $G_{T X}=$ Transmitter antenna gain $(\mathrm{dBi})$

- $G_{R X}=$ Receiver antenna gain (dBi)

- $L_{T X}=$ Transmit feeder and associated losses (e.g., feeder, connectors, etc.) (dB)

- $L_{F S}=$ Free space loss or path loss $(\mathrm{dB})$

- $L_{M}=$ Miscellaneous signal propagation losses (e.g., fading margin, polarization mismatch, medium losses etc.) $(\mathrm{dB})$

- $L_{R X}=$ Receiver feeder and associated losses (e.g., feeder, connectors, etc.) (dB)

- $S_{R X}=$ Receiver sensitivity $(\mathrm{dBm})$

In general, the standard downlink and uplink link budgets computed in Table I would apply for the both UNB-based and SS-based LPWAN system; however, the way they provide this reasonable link budget is different. UNB-based LPWAN technologies provide a high link budget by encoding the signal in an UNB (by compressing each carrier signal in around $100 \mathrm{~Hz}$ ). Having used UNB, these modulation techniques share the overall spectrum very efficiently among multiple links and increase the number of supported enddevices per unit bandwidth. SS-based LPWAN technologies

\begin{tabular}{|c|c|c|c|}
\hline Factor & $\begin{array}{c}\text { Link } \\
\text { Budget for } \\
\text { Downlink }\end{array}$ & \begin{tabular}{|c|} 
Link \\
Budget for \\
Uplink
\end{tabular} & Description \\
\hline $\begin{array}{l}\text { Transmitter } \\
\text { Power }\end{array}$ & $\begin{array}{l}500 \mathrm{~mW} \\
\text { ERP }\end{array}$ & $25 \mathrm{~mW}$ ERP & $\begin{array}{l}\text { In most country, it is } \\
\text { a regulatory limit }\end{array}$ \\
\hline ERP to EIRP & $2.15 \mathrm{~dB}$ & $2.15 \mathrm{~dB}$ & $\begin{array}{l}\text { Based on } \\
\text { Guidance }\end{array}$ \\
\hline $\begin{array}{l}\text { Transmitter } \\
\text { EIRP }\end{array}$ & $29 \mathrm{dBm}$ & $16 \mathrm{dBm}$ & $\begin{array}{l}\text { Derived from other } \\
\text { values }\end{array}$ \\
\hline $\begin{array}{l}\text { Thermal Noise } \\
\text { Density }\end{array}$ & $\begin{array}{l}-174 \mathrm{dBm} / \\
\mathrm{Hz}\end{array}$ & $\begin{array}{l}-174 \mathrm{dBm} / \\
\mathrm{Hz}\end{array}$ & $\begin{array}{l}\text { It is used as the ul- } \\
\text { timate noise floor }\end{array}$ \\
\hline Noise Figure & $8 \mathrm{~dB}$ & $5 \mathrm{~dB}$ & $\begin{array}{l}\text { Assumed within the } \\
\text { normal range }\end{array}$ \\
\hline $\begin{array}{l}\text { Receiver } \\
\text { Bandwidth/ } \\
\text { Channel }\end{array}$ & $500 \mathrm{~Hz}$ & $250 \mathrm{~Hz}$ & $\begin{array}{l}\text { Assumed within the } \\
\text { standard range }\end{array}$ \\
\hline$E_{b} / N_{0}$ & $9 \mathrm{~dB}$ & $9 \mathrm{~dB}$ & $\begin{array}{l}\text { Assumed within the } \\
\text { normal range }\end{array}$ \\
\hline Sensitivity & $-130 \mathrm{dBm}$ & $-136 \mathrm{dBm}$ & $\begin{array}{l}\text { Derived from other } \\
\text { values }\end{array}$ \\
\hline Antenna Gain & $0 \mathrm{dBi}$ & $8 \mathrm{dBi}$ & $\begin{array}{l}\text { Assumed } 0 \mathrm{dBi} \text { for } \\
\text { omni antenna and } \\
8 \mathrm{dBi} \text { for antenna at } \\
870 \mathrm{MHz}\end{array}$ \\
\hline Feeder Losses & $0 \mathrm{~dB}$ & $0 \mathrm{~dB}$ & $\begin{array}{ll}\text { Assumed standard } \\
\text { case }\end{array}$ \\
\hline $\begin{array}{l}\text { Fading and } \\
\text { Penetration } \\
\text { Losses }\end{array}$ & $0 \mathrm{~dB}$ & $0 \mathrm{~dB}$ & $\begin{array}{l}\text { Assumed AWGN } \\
\text { Channel }\end{array}$ \\
\hline $\begin{array}{l}\text { Maximum } \\
\text { Coupling Loss }\end{array}$ & $159 \mathrm{~dB}$ & $160 \mathrm{~dB}$ & $\begin{array}{l}\text { Derived from other } \\
\text { values }\end{array}$ \\
\hline
\end{tabular}

TABLE I: Computation of Link Budget for Downlink and Uplink for UNB and SS techniques for LPWAN

which use $125 \mathrm{kHz}$ bandwidth (allowing 2 channels to be used in the $250 \mathrm{kHz}$ relatively high power European subband) would have processing gains approximately $24 \mathrm{~dB}$ (downlink) or $28 \mathrm{~dB}$ (uplink).

Summary: In a nutshell, in LPWAN, managing such high path losses with limited transmit power is a difficult task, but attainable with a limited data rate. Normally, the uplink and downlink budgets are asymmetric for the long-range transmissions due to the regulatory restrictions and limited effective radiated power (ERP) [22], [23]. Both UNB and SS techniques can achieve a reasonable and similar link budget assuming the same parameter values and environment is provided as given in Table I.

\section{Coexistence}

The coexistence of several networks in LPWAN is quite common and, therefore, their coordination is crucial for uninterrupted communications. This coexistence may be of two UNBs, two SSs or a UNB and SS. The coexistence of any two networks causes two major problems mutual interference and mainly uplink blocking of both networks, which share the channel. In LPWAN, the equitable coexis- 
tence of two networks is possible only in a very low capacity deployment when very few simultaneous users are active in the interfering system.

In UNB-based LPWAN, the coexistence of two networks causes fewer problems and can amicably share an available spectrum/capacity. The interference between the systems can be reduced by using extra channels in both systems. However, this will require that base stations process extra channels but without any adverse impact on the system performance. The deployment of two uncoordinated UNB is also possible if the dimensioning process considers and resolves the possibility of interference. In SS-based LPWAN, the coexistence of two networks causes more problems than UNB networks in sharing an available spectrum/capacity. They are called bad neighbours and mitigation of the interference impact is relatively difficult.

Summary: In a nutshell, the coexistence of two networks in LPWAN causes more interference problems in the uplink than in the downlink in both techniques. The successful coexistence of two UNB networks requires frequency reassignment and dimensioning. Similarly, the successful coexistence of two SS networks requires synchronisation. The analysis of the coexistence conditions of UNB and SS networks in LPWAN and their impacts and mitigations are shown in Table II.

\section{UNB AND SS BASED LPWAN TECHNOLOGIES FOR IOT SYSTEMS AND THEIR COMPARATIVE ANALYSIS}

This section briefly describes the two most popular UNBbased LPWAN technologies Sigfox and Telensa, and two SS-based LPWAN technologies LoRa and RPMA to provide their strengths and limitations for IoT applications. Table III illustrates the comparative analysis of all these four LPWAN technologies Sigfox, Telensa, LoRa and RPMA.

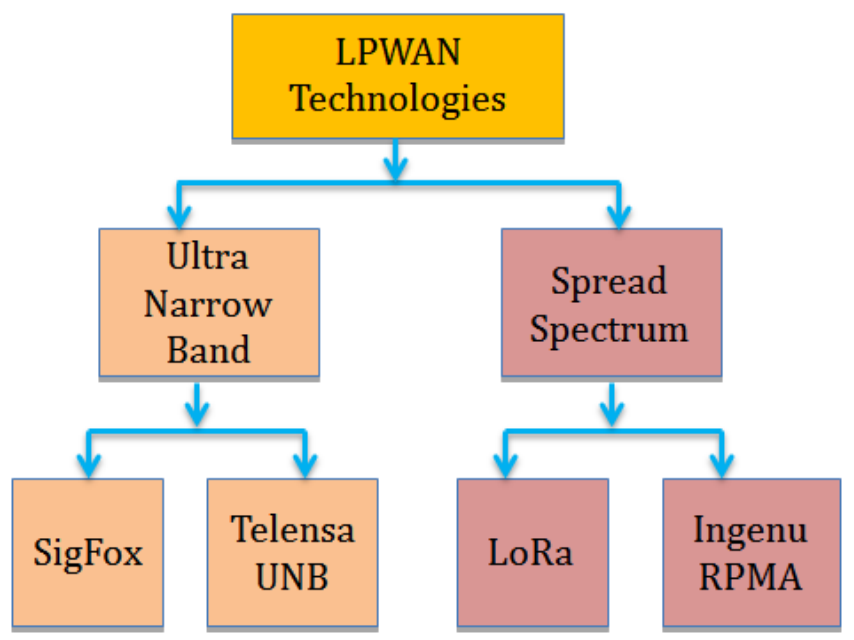

Fig. 8: UNB and SS Based LPWAN Communication Technologies for IoT Applications

Sigfox: Sigfox is a UNB-based LPWAN technology which is developed by French company Sigfox. It uses unlicensed spectrum for IoT connectivity and transmits only small amount of data. Sigfox is effectual for uplink communications from endpoints to base stations, however, it is not very effectual for downlink communications from base stations to endpoints. Sigfox is presently available in more than 25 countries worldwide.

Telensa: Telensa is another UNB-based LPWAN technology which is developed by Telensa. It uses unlicensed spectrum for IoT connectivity and transmits only small amount of data. Telensa PLANet is the most popular Central Management System (CMS) for smart streetlights used in many countries. Telensa is presently available in more than 30 countries worldwide.

LoRa: LoRa is a SS-based LPWAN technology which is developed by Semtech. Semtech builds LoRa Technology into chipsets, which are then used to build IoT products. LoRa facilitates enterprises and individuals to roll out their own IoT infrastructure using unlicensed spectrum. LoRa Alliance is an open, non-profit association, which is responsible to develop LoRa technology based protocol specification called LoRaWAN. LoRa Alliance is working in more than 100 countries.

RPMA: Random Phase Multiple Access (RPMA) is a SS-based LPWAN technology which is developed by Ingenu. It uses unlicensed ISM (Industrial, Scientific and Medical) band and offers secure communications using AES 128-bit encryption. Ingenu and u-blox are working together to deliver RPMA for IoT applications, where u-blox is making modules supporting RPMA technology. RPMA is available in more than 25 countries worldwide.

Table III shows the comparative analysis of the four LPWAN technologies Sigfox, Telensa, LoRa and RPMA. Nonetheless, this comparative analysis can be extended to cover different layers of security provided by various LPWAN technologies for IoT/M2M applications, cloud and container based software infrastructures for prompt deployment of LPWAN technologies [24], [25], [26], [27], [28]. Different LPWAN technologies offer different levels of security such as key provisioning, message confidentiality, identity protection, device or subscriber authentication, and network authentication depending on the requirements of IoT/M2M applications [29], [30], [31], [32].

\section{CONCLUSION}

This paper addressed the selection dilemma of UNB and SS by examining some of the most crucial factors that are responsible for the performance of Low Power Wide Area Network (LPWAN) technologies such as interference, capacity, link budget and coexistence. Both have interference immunity but require proper implementation to achieve that immunity. The evaluation of capacity showed that UNB offers slightly better capacity than SS due to the requirement of redundant data added in the SS coding. The link budget analysis showed that both can achieve reasonable and similar link budget depending on their perfect deployment. Finally, coexistence analysis showed that UNB is slightly more friendly than SS signals. Thus, UNB may deliver moderately better performance in terms of capacity and 
TABLE II: Coexistence of UNB and SS based LPWAN networks and their possible conditions

\begin{tabular}{|c|c|c|c|c|}
\hline $\begin{array}{l}\text { Link Type } \\
\text { (Uplink/ } \\
\text { Downlink) }\end{array}$ & $\begin{array}{l}\text { UNB (Aggressor) causing } \\
\text { interference to UNB } \\
\text { (Victim) in the same } \\
\text { Channel Bandwidth with } \\
\text { same Data Rates }\end{array}$ & \begin{tabular}{|c|} 
UNB (Aggressor) \\
causing interference to \\
SS (Victim) in the same \\
Channel Bandwidth with \\
same Data Rates
\end{tabular} & \begin{tabular}{|c|} 
SS (Aggressor) causing \\
interference to SS \\
(Victim) in the same \\
Channel Bandwidth with \\
same Data Rates
\end{tabular} & $\begin{array}{l}\text { SS (Aggressor) causing } \\
\text { interference to UNB (Vic- } \\
\text { tim) in the same Channel } \\
\text { Bandwidth with same Data } \\
\text { Rates }\end{array}$ \\
\hline $\begin{array}{l}\text { Uplink } \\
\text { Coexistence } \\
\text { of LPWAN } \\
\text { networks and } \\
\text { their Impact }\end{array}$ & $\begin{array}{l}\text { The probability of collision } \\
\text { increases if both UNBs are } \\
\text { using the same frequency } \\
\text { channels, but no interference } \\
\text { if both UNBs are using dif- } \\
\text { ferent channels. }\end{array}$ & $\begin{array}{l}\text { All active UNBs cause } \\
\text { interference, however, the } \\
\text { processing gain decreases } \\
\text { the impact of interference } \\
\text { on any one link. }\end{array}$ & $\begin{array}{l}\text { All active SSs cause inter- } \\
\text { ference, however, the pro- } \\
\text { cessing gain decreases the } \\
\text { impact of interference on } \\
\text { any one link. }\end{array}$ & $\begin{array}{l}\text { All active SSs cause interfer- } \\
\text { ence, however, the process- } \\
\text { ing gain decreases the im- } \\
\text { pact of interference on any } \\
\text { one link. }\end{array}$ \\
\hline $\begin{array}{l}\text { Uplink } \\
\text { Coexistence } \\
\text { of LPWAN } \\
\text { networks and } \\
\text { their Mitigations }\end{array}$ & $\begin{array}{l}\text { It is easy to mitigate by us- } \\
\text { ing a default retransmission } \\
\text { policy or dimensioning of } \\
\text { channels. It can also be mit- } \\
\text { igated by increasing number } \\
\text { of channels or base stations. }\end{array}$ & $\begin{array}{l}\text { It is difficult to mitigate im- } \\
\text { pact of interference caused } \\
\text { by multiple UNBs in up- } \\
\text { link. }\end{array}$ & $\begin{array}{l}\text { It can be mitigated by split- } \\
\text { ting users into near and } \\
\text { far groups on different fre- } \\
\text { quency channels; however, } \\
\text { it may not be possible for } \\
\text { dynamic end-points. }\end{array}$ & $\begin{array}{l}\text { It is difficult to mitigate im- } \\
\text { pact of interference caused } \\
\text { by multiple UNBs in uplink. } \\
\text { However, it can be mitigated } \\
\text { by coordinating the two co- } \\
\text { located base stations using } \\
\text { power control. }\end{array}$ \\
\hline $\begin{array}{l}\text { Downlink } \\
\text { Coexistence } \\
\text { of LPWAN } \\
\text { networks and } \\
\text { their Impacts }\end{array}$ & $\begin{array}{l}\text { The probability of collision } \\
\text { increases if both UNBs are } \\
\text { using the same frequency } \\
\text { channels, but no interference } \\
\text { if both UNBs are using dif- } \\
\text { ferent channels. }\end{array}$ & $\begin{array}{l}\text { All active UNBs cause } \\
\text { interference, however, the } \\
\text { processing gain decreases } \\
\text { the impact of interference } \\
\text { on any one link. }\end{array}$ & $\begin{array}{l}\text { All active SSs cause inter- } \\
\text { ference, however, the pro- } \\
\text { cessing gain decreases the } \\
\text { impact of interference on } \\
\text { any one link. }\end{array}$ & $\begin{array}{l}\text { All active SSs cause interfer- } \\
\text { ence, however, the process- } \\
\text { ing gain decreases the im- } \\
\text { pact of interference on any } \\
\text { one link. }\end{array}$ \\
\hline $\begin{array}{l}\text { Downlink } \\
\text { Coexistence } \\
\text { of LPWAN } \\
\text { networks and } \\
\text { their Mitigations }\end{array}$ & $\begin{array}{l}\text { It is easy to mitigate by us- } \\
\text { ing a default retransmission } \\
\text { policy or dimensioning of } \\
\text { channels. It can also be mit- } \\
\text { igated by increasing number } \\
\text { of channels or base stations. }\end{array}$ & $\begin{array}{l}\text { It is difficult to mitigate } \\
\text { the impact of interference } \\
\text { caused by multiple UNBs } \\
\text { in downlink, however, it is } \\
\text { less severe than uplink. }\end{array}$ & $\begin{array}{l}\text { It can be mitigated by split- } \\
\text { ting users into near and } \\
\text { far groups on different fre- } \\
\text { quency channels; however, } \\
\text { it may not be possible for } \\
\text { dynamic end-points. }\end{array}$ & $\begin{array}{l}\text { It is difficult to mitigate im- } \\
\text { pact of interference caused } \\
\text { by multiple UNBs in uplink. } \\
\text { However, it can be mitigated } \\
\text { by coordinating the two co- } \\
\text { located base stations using } \\
\text { power control. }\end{array}$ \\
\hline
\end{tabular}

TABLE III: Comparative analysis of UNB and SS based LPWAN technologies for IoT applications

\begin{tabular}{|c|c|c|c|c|}
\hline Criteria & Sigfox & Telensa & LoRa & RPMA \\
\hline 1. Transmission Range & <13km (Approximately) & $<10 \mathrm{~km}$ (Approximately) & $<15 \mathrm{~km}$ (Approximately) & $<15 \mathrm{~km}$ (Approximately) \\
\hline $\begin{array}{l}\text { 2. Maximum Coupling } \\
\text { Loss / Coverage }\end{array}$ & $149 \mathrm{~dB}$ & $154 \mathrm{~dB}$ & $157 \mathrm{~dB}$ & $177 \mathrm{~dB}$ \\
\hline 3. Licensing & Unlicensed & Unlicensed & Unlicensed & Unlicensed \\
\hline 4. Model & Proprietary & Proprietary & Alliance & Proprietary \\
\hline $\begin{array}{l}\text { 5. Spectrum / } \\
\text { Frequency Band }\end{array}$ & $\begin{array}{l}\text { Sub-GHz ISM/ TV-White } \\
\text { Spaces }\end{array}$ & $\begin{array}{l}\text { Sub-GHz ISM/TV-White } \\
\text { Spaces }\end{array}$ & Sub-GHz ISM & ISM $2.4 \mathrm{GHz}$ \\
\hline 6. Bandwidth & $100 \mathrm{~Hz} / 900 \mathrm{MHz}, 100 \mathrm{~Hz}$ & $868 \mathrm{MHz}, 915 \mathrm{MHz}$ & $\begin{array}{l}125 \mathrm{kHz} \\
<500 \mathrm{kHz}\end{array}$ & $1 \mathrm{MHz} / 2.4 \mathrm{GHz}$ \\
\hline $\begin{array}{l}\text { 7. Capacity/ } \\
\text { Connections } \\
\end{array}$ & $50,000 /$ cell & $500,000 /$ server & $40,000 /$ cell & $500,000 /$ cell \\
\hline 8. Battery Life & 10years+ & 10years+ & 10years+ & 10years+ \\
\hline 9. Data Rate & $<100$ bps & $<100$ bps & $<10 \mathrm{kbps}$ & $<624 \mathrm{kbps}$ \\
\hline 10. Modulation & $\begin{array}{ll}\text { UNB/ } & \text { BPSK(UL)/ } \\
\text { GFSK(DL) } & \end{array}$ & UNB 2-FSK & CSS & $\begin{array}{l}\text { RPMA-DSSS(UL), } \\
\text { CDMA(DL) }\end{array}$ \\
\hline 11. Bi-directional & No & Yes & Dependent on the Class & Yes \\
\hline 12. Handover/Handoff & No & Yes & No & Yes \\
\hline 13. Global Ecosystem & Weak & Weak & Fragmented by Region & Worldwide Network \\
\hline 14. Location Support & No & No & Yes & No/Requires GPS \\
\hline 15. IoT Applications & $\begin{array}{llc}\text { Smart Cities, Smart } \\
\text { Alarms, }\end{array}$ & \begin{tabular}{|llr} 
Smart & Cities, & Smart \\
Lighting, & Smart & Parking \\
\end{tabular} & $\begin{array}{l}\text { Smart Cities, Smart build- } \\
\text { ing, Smart Meters }\end{array}$ & $\begin{array}{l}\text { Smart Cities, Smart Meters, } \\
\text { Smart Grid, Smart Lighting }\end{array}$ \\
\hline
\end{tabular}


coexistence based on its flawless implementation. However, this is difficult to achieve in practice; additionally SS may offer better security, speed and accuracy features, which are not considered in this evaluation. Therefore, it requires further in-depth evaluation by considering some additional criteria.

\section{REFERENCES}

[1] N. Naik, "Choice of effective messaging protocols for IoT systems: MQTT, CoAP, AMQP and HTTP," in 2017 IEEE International Systems Engineering Symposium (ISSE). IEEE, 2017.

[2] Gsma.com. (2017) Low Power Wide Area Network to lead IoT connections by 2022. [Online]. Available: https://www.gsma.com/newsroom/press-release/low-powerwide-area-networks-lead-iot-connections-2022/

[3] T. Rebbeck, M. Mackenzie, and N. Afonso, "Low-powered wireless solutions have the potential to increase the $\mathrm{m} 2 \mathrm{~m}$ market by over 3 billion connections," Analysys Mason, 2014.

[4] M. Anteur, V. Deslandes, N. Thomas, and A.-L. Beylot, "Ultra narrow band technique for low power wide area communications," in IEEE Global Communications Conference (GLOBECOM), 2015, pp. 1-6.

[5] H. Walker, "Ultra narrow band modulation," in IEEE/Sarnoff Symposium on Advances in Wired and Wireless Communication, 2004, pp. $19-22$.

[6] X. Xiong, K. Zheng, R. Xu, W. Xiang, and P. Chatzimisios, "Low power wide area machine-to-machine networks: Key techniques and prototype," IEEE Communications Magazine, vol. 53, no. 9, pp. 6471, 2015.

[7] S. Kartakis, B. D. Choudhary, A. D. Gluhak, L. Lambrinos, and J. A. McCann, "Demystifying low-power wide-area communications for city iot applications," in Proceedings of the Tenth ACM International Workshop on Wireless Network Testbeds, Experimental Evaluation, and Characterization. ACM, 2016, pp. 2-8.

[8] U. Raza, P. Kulkarni, and M. Sooriyabandara, "Low power wide area networks: An overview," IEEE Communications Surveys \& Tutorials, vol. 19, no. 2, pp. 855-873, 2017.

[9] Ni.com. (2014) Understanding spread spectrum for communications. [Online]. Available: http://www.ni.com/white-paper/4450/en/

[10] N. Naik and P. Jenkins, "Web protocols and challenges of web latency in the web of things," in Ubiquitous and Future Networks (ICUFN), 2016 Eighth International Conference on. IEEE, 2016, pp. 845-850.

11] N. Naik, P. Jenkins, P. Davies, and D. Newell, "Native web communication protocols and their effects on the performance of web services and systems," in Computer and Information Technology (CIT), 2016 IEEE International Conference on. IEEE, 2016, pp. 219-225.

[12] M2comm. (2017) WAN - Ultra-Narrow Band (UNB). [Online]. Available: http://www.m2comm.co/front-page/technology/ wan-ultra-narrow-band-unb/

[13] T. Lassen, "Long-range RF communication: Why narrowband is the de facto standard" in White Paper, 2014

[14] H. R. Walker, "Understanding ultra narrowband modulation," $M i-$ crowaves \& $R F$, vol. 42 , no. 12 , pp. 53-62, 2003.

[15] R. L. Peterson, R. E. Ziemer, and D. E. Borth, Introduction to spreadspectrum communications. Prentice Hall New Jersey, 1995, vol. 995.

[16] P. Prabakaran. (2003) Tutorial on spread spectrum technology. [Online]. Available: http://www.eetimes.com/document.asp?doc_id= 1271899

[17] J. Meel, "Spread spectrum (SS)," De Nayer Instituut, Hogeschool Voor Wetenschap \& Kunst, 1999.

[18] J. Burns, S. Kirtay, and P. Marks. (2015, July 14) Future use of licence exempt radio spectrum : A reportfor the uk spectrum policy forum. [Online]. Available: http://www.plumconsulting.co.uk/pdfs/Plum July 2015 Future use_of_Licence_Exempt_Radio_Spectrum.pdf

[19] Comtechefdata.com. (2012) Spread Spectrum in the SLM5650A: Features, performance, and applications. [Online]. Available: http://www.comtechefdata.com/files/articles_papers/WPSpread-Spectrum-in-SLM-5650A.pdf

[20] RealWireless. (2015) A comparison of UNB and spread spectrum wireless technologies as used in LPWA M2M applications. [Online]. Available: https://www.thethingsnetwork.org/forum/uploads/default/ original/1X/3b1c1ae4a925e9aa897110ccde10ec61f3106b87.pdf
21] J.-P. Bardyn, T. Melly, O. Seller, and N. Sornin, "IoT: The era of LPWAN is starting now," in European Solid-State Circuits Conference, ESSCIRC Conference 2016: 42nd. IEEE, 2016, pp. 25-30.

[22] S. Andreev, O. Galinina, A. Pyattaev, M. Gerasimenko, T. Tirronen, J. Torsner, J. Sachs, M. Dohler, and Y. Koucheryavy, "Understanding the iot connectivity landscape: a contemporary $\mathrm{m} 2 \mathrm{~m}$ radio technology roadmap," IEEE Communications Magazine, vol. 53, no. 9, pp. 3240, 2015

[23] M. Lauridsen, H. Nguyen, B. Vejlgaard, I. Kovacs, P. Mogensen, and M. Sørensen, "Coverage comparison of GPRS, NB-IoT, LoRa, and SigFox in a $7800 \mathrm{~km} 2$ area," in IEEE Vehicular Technology Conference, 2017.

[24] N. Naik, "Migrating from virtualization to dockerization in the cloud: Simulation and evaluation of distributed systems," in IEEE 10th International Symposium on the Maintenance and Evolution of Service-Oriented and Cloud-Based Environments, (MESOCA). IEEE, 2016, pp. 1-8.

[25] _ _ "Building a virtual system of systems using Docker Swarm in multiple clouds," in IEEE International Symposium on Systems Engineering (ISSE 2016). IEEE, 2016.

[26] - "Connecting Google cloud system with organizational systems for effortless data analysis by anyone, anytime, anywhere," in IEEE International Symposium on Systems Engineering (ISSE ). IEEE, 2016

[27] — "Applying computational intelligence for enhancing the dependability of multi-cloud systems using docker swarm," in IEEE Symposium Series on Computational Intelligence (SSCI), 2016.

[28] _ "Docker container-based big data processing system in multiple clouds for everyone," in 2017 IEEE International Systems Engineering Symposium (ISSE), 2017.

[29] N. Naik and P. Jenkins, "A secure mobile cloud identity: Criteria for effective identity and access management standards," in 2016 4th IEEE International Conference on Mobile Cloud Computing, Services, and Engineering (MobileCloud 2016). IEEE, 2016.

[30] _ _ "An analysis of open standard identity protocols in cloud computing security paradigm," in 14th IEEE International Conference on Dependable, Autonomic and Secure Computing (DASC 2016). IEEE, 2016

[31] - "Securing digital identities in the cloud by selecting an apposite federated identity management from saml, oauth and openid connect," in 2017 11th International Conference on Research Challenges in Information Science (RCIS). IEEE, 2017, pp. 163-174.

[32] N. Naik, P. Jenkins, and D. Newell, "Choice of suitable identity and access management standards for mobile computing and communication," in 2017 24th International Conference on Telecommunications (ICT). IEEE, 2017, pp. 1-6. 